\title{
Huge Saccular Aneurysm in a Coronary-Pulmonary Fistula Fed by the Left and Right Coronary Arteries
}

\author{
Keiji Hirooka, MD; Akihisa Hanatani, MD*; Satoshi Nakatani, MD*; \\ Yoshio Yasumura, MD*; Ko Bando, MD**; \\ Kunio Miyatake, MD*; Masakazu Yamagishi, MD*
}

\begin{abstract}
A 62-year-old woman was referred because of an abnormal mediastinal shadow that had first been noted 4 years ago on the left cardiac border on plain chest roentgenogram and which had gradually increased in size since. A huge saccular aneurysm in coronary-pulmonary fistula into which both the left and right coronary arteries drained was revealed by computed tomography and coronary angiography. The patient underwent aneurysmal resection and ligation of the fistulas. Histological study of the aneurysmal wall did not reveal atherosclerotic change but instead cystic medial necrosis, although there was no other clinical manifestation associated with this. (Circ J 2002; 66: 525-527)
\end{abstract}

Key Words: Coronary-pulmonary fistula; Saccular aneurysm; Surgical resection

B cause a communication between the coronary arteries and the cardiac chamber or the pulmonary trunk is not a common anomaly, the formation of an aneurysm in this communication vessel is clinically few? $?^{-4}$ We report an adult case of a huge aneurysm that was fed by both the left anterior descending and the right coronary artery draining anomalously into the main pulmonary artery.

\section{Case Report}

A 62-year-old woman was referred to hospital because of an abnormal mediastinal shadow on chest roentgenogram. She had been pointed out a heart murmur 30 years ago, but did not have further examinations because she did not have any symptoms. An abnormal shadow on the left cardiac border was first noted on plain chest roentgenogram 4 years ago and since then the size of the mass had gradually increased (Fig 1). However, she did not develop angina-like chest pain on effort.

Physical examination revealed a grade $4 / 6$ continuous cardiac murmur at the second intercostal space. Blood pressure was $135 / 80 \mathrm{mmHg}$. There was complete right bundle branch block on ECG. Two-dimensional echocardiography revealed a round cystic lesion with internal spontaneous echo contrast, located in contact with the pulmonary trunk (Fig 2). Computed tomography showed a giant mass of $5.8 \times 6.0 \mathrm{~cm}$ in diameter into which the left and right coronary arteries drained (Fig 3). During catheterization, oximetry revealed an oxygen step-up of $4 \%$ between the pulmonary artery and the right ventricle, consistent with a left-to-right shunt of 3.4\%. The pulmonic-to-systemic blood flow ratio $(\mathrm{Qp} / \mathrm{Qs})$ was 1.04 . Digital subtraction coronary

(Received May 18, 2001; revised manuscript received June 22, 2001; accepted July 4, 2001)

Cardiovascular Division, Osaka National Hospital and *Cardiology Division of Medicine and **Cardiovascular Surgery, National Cardiovascular Center, Osaka, Japan

Mailing address: Keiji Hirooka, MD, Cardiovascular Division, Osaka National Hospital, 2-1-14 Hoenzaka, Chuo-ku, Osaka 5400006, Japan. E-mail: k-chan@onh.go.jp angiography confirmed that each branch of the left anterior descending artery and the right coronary artery drained into the huge aneurysm (Fig 4). However, the efferent vessels into the pulmonary artery were not clearly demonstrated by angiography, but color Doppler flow imaging revealed the small efferent flow was running from the aneurysm to the main pulmonary artery (Fig 5). From these findings, she was diagnosed as having a coronary-pulmonary fistula with a concomitant huge saccular aneurysm that was considered to have progressively enlarged during the preceding 4 years, and surgical resection was proposed.

At operation, an egg-shaped, elastic, cardiac tumor $6 \mathrm{~cm}$ in diameter was found in front of the right ventricular outflow tract. The afferent vessels were confirmed to arise

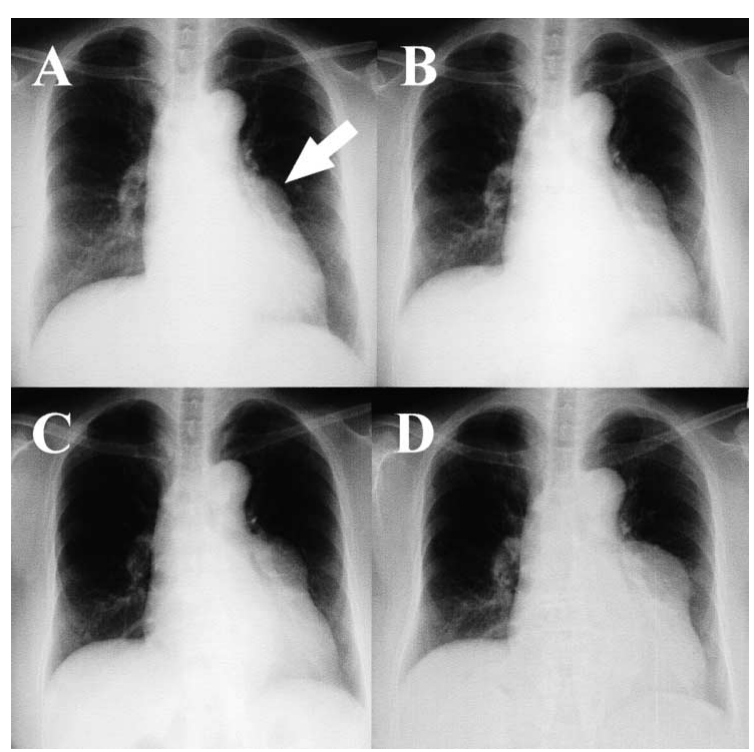

Fig 1. Serial chest roentgenograms show the gradually expanding left cardiac border (white arrow). (A) 4 years earlier, (B) 2 years earlier, (C) last year, (D) on admission. 


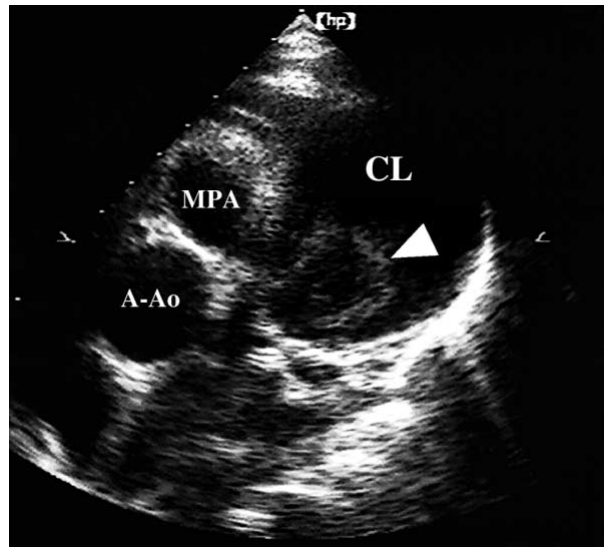

Fig 2. Two-dimensional echocardiography shows a round cystic lesion (CL) with spontaneous echo contrast inside (white arrowhead), in contact with the main pulmonary artery (MPA). A-Ao, ascending aorta.

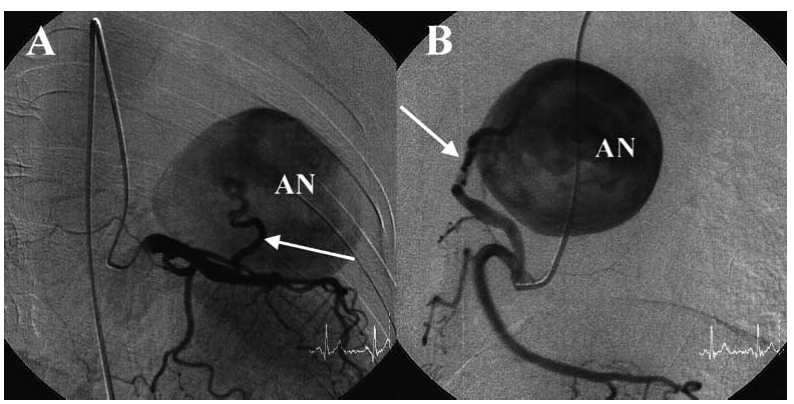

Fig 4. Digital subtraction coronary angiography. The fistulous vessels arising from the proximal sites of the left anterior descending artery (LAD; A) and RCA (B) drain into the huge aneurysm (white arrows). However, the efferent vessel to the pulmonary artery are not shown. Abbreviations as in Fig 3.

from the left anterior descending artery and the right coronary artery. There was a single efferent hole of $5 \mathrm{~mm}$ in diameter in contact with the main pulmonary artery. Under cardiopulmonary bypass, the afferent and efferent vessels were individually ligated, and the aneurysm was resected. Histological study of the aneurysmal wall revealed not atherosclerotic change, but rather cystic medial necrosis, although there was no other clinical manifestation associated with the medial necrosis. Postoperative coronary angiography revealed no evidence of the aneurysm or the feeding arteries, and the continuous cardiac murmur had disappeared.

\section{Discussion}

Coronary arteriovenous fistula is an uncommon congenital cardiac malformation identified in only $0.1 \%$ of routine cardiac angiographic studies! With regard to the vessel of origin, $50 \%$ arose from the right coronary artery, $42 \%$ from the left coronary artery, $5 \%$ from both vessels and $3 \%$ were not specified5 Drainage occurred into the right ventricle in $41 \%$, into the right atrium in $26 \%$, into the pulmonary artery in $17 \%$, into the coronary sinus in $7 \%$, into the left atrium in $5 \%$, into the left ventricle in $3 \%$ and into the superior vena cava in $1 \% 5$

There are previous reports of cases of aneurysms associ-

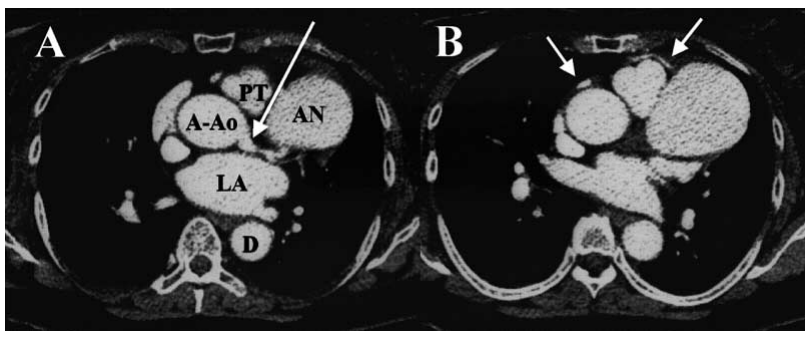

Fig 3. Computed tomography images. A giant mass is located to the left of the pulmonary trunk (PT). The afferent vessels arising from the left coronary artery (LCA; A) and right coronary artery (RCA; B) drain into the mass lesion (white arrows). D, descending aorta; AN, aneurysm; LA, left atrium; other abbreviations as in Fig 2.

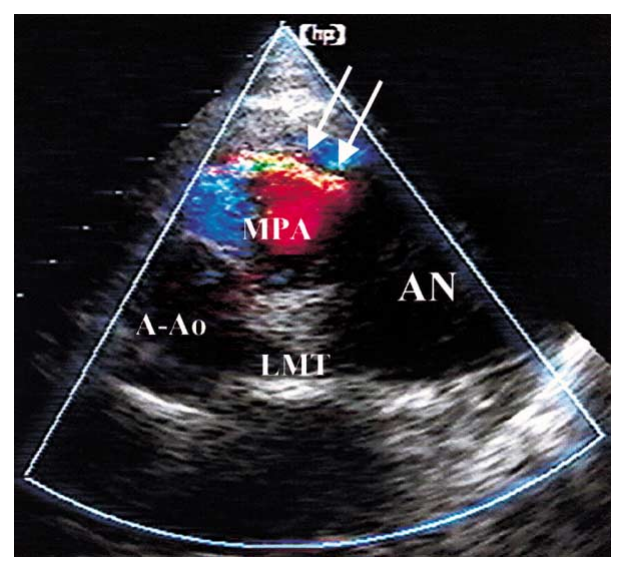

Fig 5. Doppler echocardiography. Color Doppler image visualizes the small efferent flow from the aneurysm to the main pulmonary artery (white arrows). LMT, left main trunk; other abbreviations as in Figs 2 and 3.

ated with coronary artery fistulas. Urrutia et al reported that multiple fistulas were identified in $16 \%$ of 58 patients and aneurysmal fistula in $19 \%$, but most of these are fusiform aneurysms, and saccular aneurysms are extremely rare?,4 To our knowledge, this is the first report of a huge saccular aneurysm of a coronary arteriovenous fistula that drained from multiple coronary arteries.

Coronary arteriovenous fistula develop either from enlargement of the capillary network during cardiac embryogenesis or when the main coronary arteries remain attached to instead of separating from the pulmonary trunk6 Although the exact mechanism by which the 2 fistulous vessels drained into the aneurysm remains unknown, it is possible that separately developing aneurysms in each coronary artery fused together or both feeding arteries might have merged into a single vessel where the aneurysm occurred. Generally, the cause of saccular aneurysm concomitant with coronary arteriovenous fistula is suspected to be inflammation, injury or stenosis with atherosclerotic change? In the present case, the patient had never experienced chest injury, and histology could not prove a relationship between the aneurysm and atherosclerotic change.

The management of coronary arteriovenous fistula is still controversial, especially regarding operative intervention in asymptomatic patients ${ }^{8-10}$ In this case, progressive dilatation of the aneurysm to a diameter exceeding $5 \mathrm{~cm}$ suggested that the possibility of acute rupture was an indication for surgical resection, despite the small shunt ratio 
and lack of clinical symptoms.

\section{References}

1. Vavuranakis M, Bush CA, Boudouls H. Coronary artery fistulas in adults: Incidence, angiographic characteristics, natural history. Cathet Cardiovasc Diagn 1995; 35: 116-120.

2. Urrutia SCO, Falaschi G, Ott DA, Cooley DA. Surgical management of 56 patients with congenital coronary artery fistulas. Ann Thorac Surg 1983; 35: 300-307.

3. Okita Y, Miki S, Kusuhara K, Ueda Y, Tahata T, Sakai T, et al. Aneurysm of coronary arteriovenous fistula presenting as a calcified mediastinal mass. Ann Thorac Surg 1992; 54: 771-773.

4. Tayama E, Ohashi M, Fukunaga S, Hayashida N, Akashi H, Kawara $\mathrm{T}$, et al. Surgical treatment of a coronary artery fistula with concomitant saccular coronary artery aneurysm: A case report. Jpn Circ J
1999; 63: 809-812.

5. Levin DC, Fellows KE, Abrams HL. Hemodynamically significant primary anomalies of the coronary arteries: Angiographic aspects. Circulation 1978; 58: 25-34.

6. Dervan JP, Vlay SC. Coronary artery-to-pulmonary artery fistula: A potential new mechanism. Am Heart J 1989; 117: 971-973.

7. Miyauchi Y, Uemura K, Goto H, Tanaka M, Kawaguchi H, Tadakuma $\mathrm{K}$, et al. A case of coronary artery-pulmonary artery fistula with a giant saccular aneurysm. Jpn J Thorac Surg 1985; 38: 408-411.

8. Rittenhouse EA, Doty DB, Ehrenhaft JL. Congenital coronary arterycardiac chamber fistula: Review of operative management. Ann Thorac Surg 1975; 20: 468-485.

9. Jaffe RB, Glancy DL, Epstein SE, Brown BG, Morrow AG. Coronary arterial-right heart fistulae. Long-term observations in seven patients. Circulation 1973; 47: 133-143.

10. Shubrooks SJ Jr, Naggar CZ. Spontaneous near closure of coronary artery fistula. Circulation 1978; 57: 197-199. 